\title{
One-Step Controllable Synthesis for Ultrafine Metal oxide Semiconductor Nanocrystals via a Separated Two-Phase Hydrolysis Reaction
}

\section{Supporting Online Information}

\author{
Kangjian Tang, Yangdong Wang, Weimin Yang, Zaiku Xie, Taolei Sun \\ and Harald Fuchs \\ Physikalisches Institut, WWU Muenster, 48149, Muenster, Germany; Center \\ for Nanotechnology, 48151, Muenster, Germany; Shanghai Research Institute \\ of Petrochemical Technology, SINOPEC, 201208, Shanghai, P. R. China.
}

sunt@uni-muenster.de

\section{Experimental Details:}

In a typical synthesis, about $9 \mathrm{~mL}$ water was added into a Teflon lined stainless steel autoclave. Subsequently, $1.0 \mathrm{~g}$ of tetra-n-butyl zirconate (TBZ) was first dissolved in $4 \mathrm{~mL}$ of toluene to serve as an organic phase. Then this solution was transferred into the inner container which was placed in a Teflon line above the surface of water. The corresponding molar ratio of $\mathrm{Zr}$ : Toluene $: \mathrm{H}_{2} \mathrm{O}$ was about 3 : $86: 1000$. The autoclave was then sealed and placed in a preheated oven and heated statically at $100-240{ }^{\circ} \mathrm{C}$ for 24 hours. After cooling, the solid product was separated by centrifugation and further washed adequately with anhydrous ethanol. The preparation of $\mathrm{TiO}_{2}$ nano-crystals was the same as of $\mathrm{ZrO}_{2}$ qdots, with a precusor of tetra-n-butyl titanate.

The photocatalytic hydrogen production experiments were carried out in a gas-closed circulation system. The photo-catalyst powder $(0.1 \mathrm{~g})$ was dispersed in aqueous/methanol solutions (420 mL, volume ratio 20/1) in an inner irradiation quartz reaction cell. The light sources were a $350 \mathrm{~W}$ high pressure Hg lamp. The amounts of $\mathrm{H}_{2}$ and $\mathrm{O}_{2}$ evolved were determined by gas chromatography. (Agilent GC-6820 TCD, $\mathrm{Ar}$ carrier). Experiments for all the samples $\left(\mathrm{ZrO}_{2}\right.$ qdots, commercial $\mathrm{ZrO}_{2}$ (Beijing Chemical Plant, China), and $\mathrm{TiO}_{2}$ (p25, Degussa)) were carried out under the same conditions. 
X-ray powder diffraction and Small angle X-ray scattering analyses of the products were carried out on a Rigaku D/MAX-2550 X-ray diffractometer with $\mathrm{CuK} \alpha$ radiation $(\lambda=1.5418 \AA ; 40 \mathrm{kV}, 200 \mathrm{~mA})$. The TEM and HRTEM analyses of the as-prepared $\mathrm{ZrO}_{2}$ nanocrystals were performed on JEOL JEM-3010. The liquid nuclear magnetic resonance was carried on Brucker Avance-500. The specific surface areas of the samples were measured by the BET method. The fluorescent properties were investigated on a Perkinelmer 1s55 luminescence spectrometer.
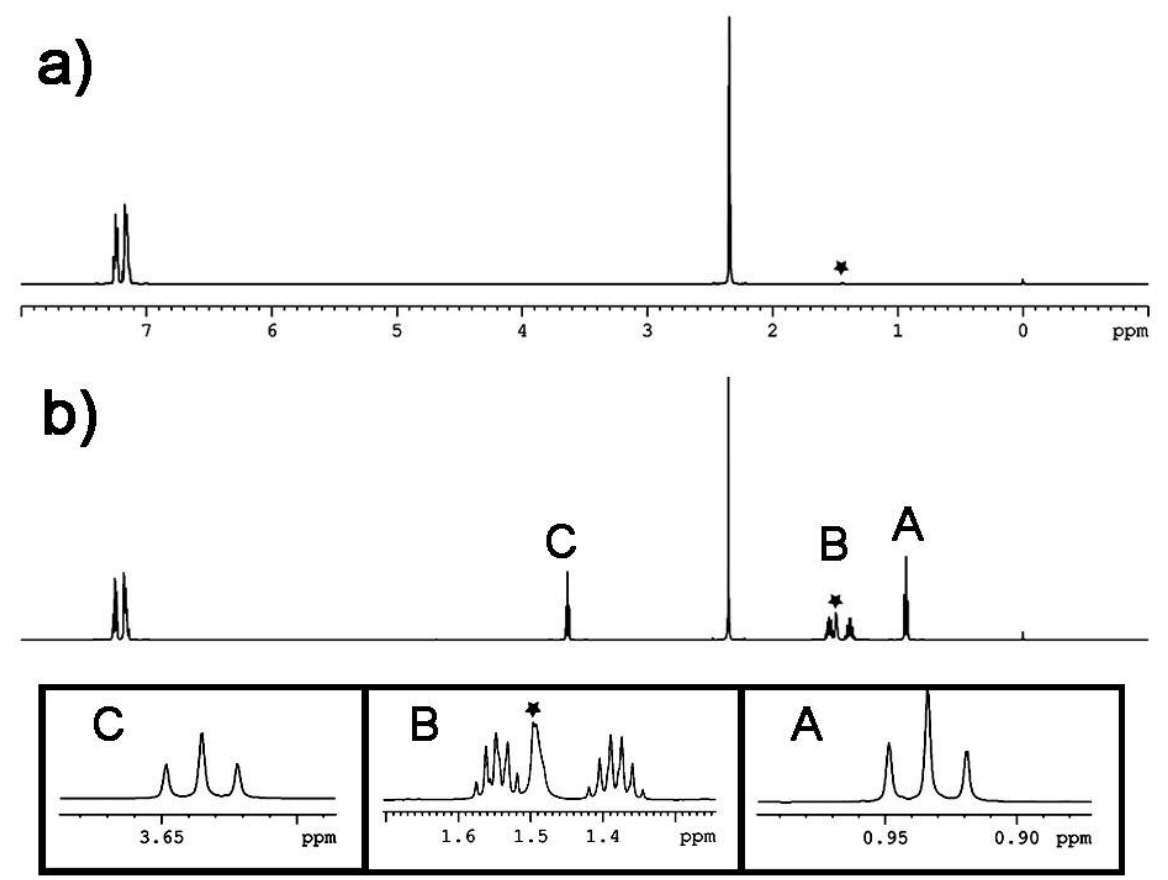

Figures S1. NMR analyses. a) ${ }^{1} \mathrm{H}$ NMR spectra of toluene; b) ${ }^{1} \mathrm{H}$ NMR spectra of solution after reaction. insert: 3 split peaks indicating a $-\mathrm{CH}_{2}$ connecting an -OH (A). two groups of 5 split peaks indicating a $-\mathrm{CH}_{2}-\mathrm{CH}_{2}-(\mathrm{B}) ; 3$ split peaks indicating a $-\mathrm{CH}_{3}$ connecting a $-\mathrm{CH}_{2}$ (C); The peak marked with a star symbol indicates an impurity in toluene. 
The scattering amplitude of SAXS is calculated according to Born approximation.

$$
A(\boldsymbol{q})=\int_{V} \Delta \rho(\boldsymbol{r}) e^{i \boldsymbol{q} \cdot r} d \boldsymbol{r} \quad q=\frac{4 \pi}{\lambda} \sin \frac{2 \theta}{2}
$$

Where, $\mathrm{q}$ is the scattering vector, $\Delta \rho$ is the electron density difference at position, $V$ is the target volume for the integral over the entire medium, and $\lambda$ is the $\mathrm{X}$-ray wavelength. $\mathrm{ZrO}_{2}$ qdots are sphere with a radius of $\mathrm{R}$, equation 1 can be deduced to the well known form:

$$
\begin{aligned}
F^{\text {sphere }}(q, D) & =\Delta \rho \cdot \int_{0}^{2 \pi} d \varphi \int_{0}^{\pi} d \theta \int_{0}^{R} e^{i q r \cos \theta} r^{2} \sin \theta d r \\
& =\Delta \rho \cdot \frac{4 \pi}{q^{3}}(\sin (q R)-q R \cos (q R))
\end{aligned}
$$

Then the particle size is obtained by calculation through the software of Nano-solver (Rigaku corporation)
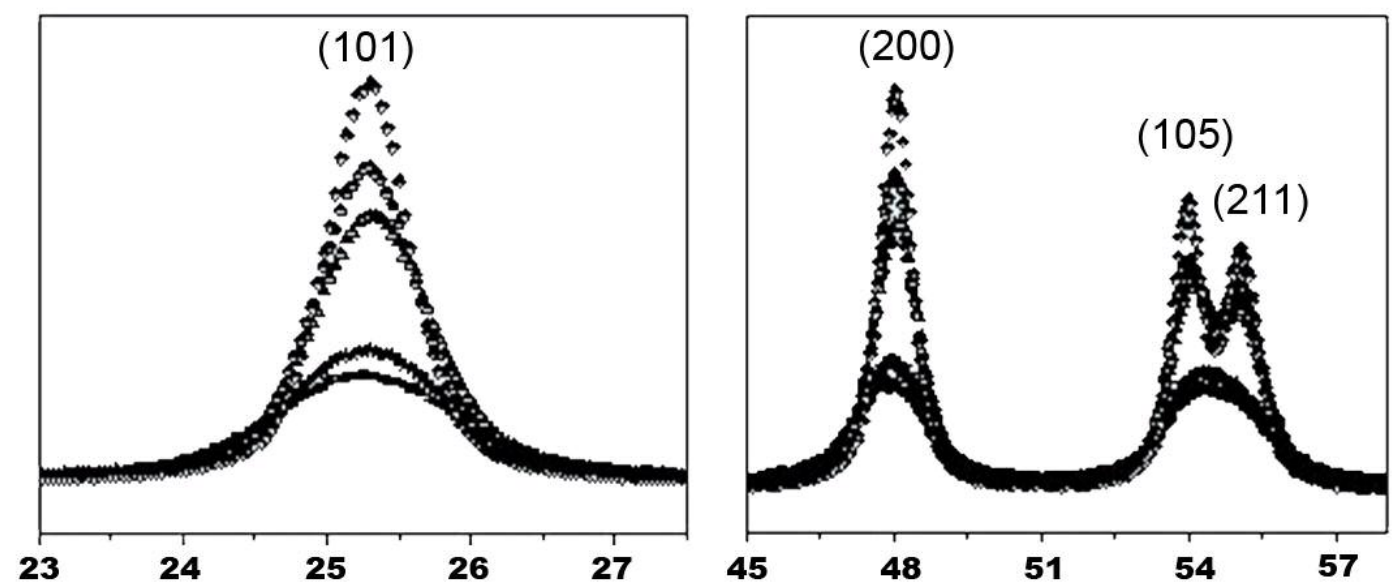

$2 \theta$

Figure S2. XRD peaks of (101), (200), (105) and (211) of $\mathrm{TiO}_{2}$ nanocrystals obtained under different temperature. The peaks were chosen to perform X-ray profile analysis. 